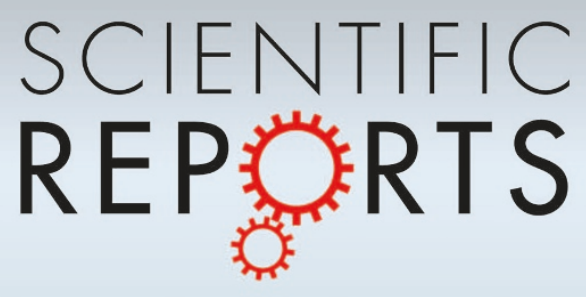

OPEN

SUBJECT AREAS:

PROTEOMICS

INDUSTRIAL MICROBIOLOGY

Received

27 June 2014

Accepted

10 October 2014

Published

7 November 2014

Correspondence and requests for materials should be addressed to L.L. (longliu@jiangnan. edu.cn) or G.C.D. lgcdu@jiangnan.edu.

cn)

\section{Understanding of how Propionibacterium acidipropionici respond to propionic acid stress at the level of proteomics}

Ningzi Guan ${ }^{1,2}$, Hyun-dong Shin ${ }^{3}$, Rachel R. Chen ${ }^{3}$, Jianghua Li Li, ${ }^{1,}$ Long Liu ${ }^{1,4}$, Guocheng Du 4 \& Jian Chen ${ }^{4}$

${ }^{1}$ Key Laboratory of Carbohydrate Chemistry and Biotechnology, Ministry of Education, Jiangnan University, Wuxi 214122, China, ${ }^{2}$ Key Laboratory of Industrial Biotechnology, Ministry of Education, Jiangnan University, Wuxi 214122, China, ${ }^{3}$ School of Chemical and Biomolecular Engineering, Georgia Institute of Technology, Atlanta 30332, USA, ${ }^{4}$ Synergetic Innovation Center OfFood Safety and Nutrition, Wuxi 214122, China.

Propionic acid (PA) is an important platform chemical in the food, agriculture, and pharmaceutical industries and is mainly biosynthesized by propionibacteria. Acid tolerance in PA-producing strains is crucial. In previous work, we investigated the acid tolerance mechanism of Propionibacterium acidipropionici at microenvironmental levels by analyzing physiological changes in the parental strain and three PA-tolerant mutants obtained by genome shuffling. However, the molecular mechanism of PA tolerance in $P$. acidipropionici remained unclear. Here, we performed a comparative proteomics study of $P$. acidipropionici CGMCC 1.2230 and the acid-tolerant mutant $P$. acidipropionici WSH1105; MALDI-TOF/ MS identified 24 proteins that significantly differed between the parental and shuffled strains. The differentially expressed proteins were mainly categorized as key components of crucial biological processes and the acid stress response. Quantitative reverse transcriptase polymerase chain reaction (qRT-PCR) was used to confirm differential expression of nine key proteins. Overexpression of the secretory protein glyceraldehyde-3-phosphate dehydrogenase and ATP synthase subunit $\alpha$ in Escherichia coli BL21 improved PA and acetic acid tolerance; overexpression of NADH dehydrogenase and methylmalonyl-CoA epimerase improved PA tolerance. These results provide new insights into the acid tolerance of $P$. acidipropionici and will facilitate the development of PA production through fermentation by propionibacteria.

ropionic acid (PA) is an important building block in organic synthesis, food, feedstuffs, perfume, paint, and pharmaceuticals ${ }^{1}$. PA is mainly produced by petrochemical synthesis. With increasing concerns about environmental pollution and energy shortages, there is growing interest in propionibacterial production of $\mathrm{PA}^{2,3}$. The highest reported PA production by bacteria was $136 \mathrm{~g} / \mathrm{L}$, obtained from constant fed-batch fermentation in a plant fibrous-bed bioreactor with immobilized Propionibacterium freudenreichii CCTCC M207015 ${ }^{4}$. Despite these achievements, industrial-scale PA production has not been achieved due to the high production cost in comparison to traditional petrochemical-based production methods.

Microbial PA production is a typical product-inhibited process, and the severe inhibition of PA on cell growth and PA synthesis limits production. Enhancement of PA tolerance is an effective strategy to alleviate inhibition and improve PA production ${ }^{2,3}$. For example, fed-batch fermentation of $P$. acidipropionici mutants obtained by adaptation in a fibrous-bed bioreactor produced $71.8 \mathrm{~g} / \mathrm{L} \mathrm{PA}, 37.5 \%$ greater than the yield from the wild-type strain $^{2}$. In previous work, we improved the acid tolerance of P. acidipropionici CGMCC 1.2230 by genome shuffling via inactivated protoplast fusion. The process yielded 3 PA-tolerant strains: $P$. acidipropionici WSH1 103 (tolerance to $10 \mathrm{~g} / \mathrm{L}$ PA), P. acidipropionici WSH1 104 (tolerance to $15 \mathrm{~g} / \mathrm{L} \mathrm{PA}$ ), and P. acidipropionici WSH1105 (tolerance to $20 \mathrm{~g} / \mathrm{L} \mathrm{PA})^{5}$. To understand the mechanism and factors contributing to enhanced acid tolerance, the Yang group characterized the morphology of cells adapted in a fibrous-bed bioreactor ${ }^{2,3}$. The mutant strains were greater in length and smaller in diameter, giving them a higher surface area and making them more efficient in transporting substrates and metabolites across the cell membrane. Increased $\mathrm{H}^{+}$-ATPase activity in the mutant indicated greater proton pumping efficiency, which may improve PA tolerance. In addition, increased long-chain saturated fatty acids (C 17:0) and fewer unsaturated fatty acids (C 18:1) reduced membrane fluidity, which may also contribute to increased PA tolerance ${ }^{2,3}$. We recently investigated the acid-tolerance mechanism of $P$. acidipropionici at the microenvironmental level by comparing the physiological changes in wild-type P. acidipropionici and three mutants; we found that the arginine deaminase (ADI) and glutamate 
decarboxylase (GAD) systems are important for acid resistance in $P$. acidipropionici ${ }^{6}$. Despite these efforts, current understanding of the acid-tolerant mechanism of $P$. acidipropionici remains limited to the microenvironmental level, and it is not clear how $P$. acidipropionici responds to acid stress at the molecular level. This level of understanding is crucial, however, for engineering $P$. acidipropionici strains with improved acid tolerance and PA production.

To deepen our understanding of the adaptive response mechanism of $P$. acidipropionici to PA stress, we used comparative proteomics to characterize the differences between wild-type $P$. acidipropionici and the acid-tolerant mutant. First, 2-dimensional electrophoresis (2-DE)-based comparative proteomics was used to characterize the acid-tolerant mutants and identify differences in protein abundance relative to the parent strain. Proteins with significantly different expression patterns were verified at the transcriptional level by quantitative reverse-transcriptase polymerase chain reaction (qRT-PCR). Five key proteins were overexpressed in Escherichia coli BL21(DE3) to verify their ability to improve its tolerance to PA and acetic acid. The results deepen our understanding of the response of $P$. acidipropionici to PA stress and make it feasible to improve acid tolerance and PA production by direct genetic manipulation.

\section{Results}

Proteome analysis and protein identification. To better understand the molecular mechanism of $P$. acidipropionici acid tolerance, we used comparative proteomics to identify differential protein expression in $P$. acidipropionici CGMCC 1.2230 and its evolved mutant $P$. acidipropionici WSH1105 based on twodimensional gel electrophoresis followed by protein identification. The gel maps (Figure S1) were analyzed with PDQuest 8.0.1. After optimization of the 2-DE gels, about 800 discrete intracellular protein spots were detected per sample. Proteins that differed by more than 2-fold were labeled in the preparative gel (Figure 1) and protein identities are listed in Table 1 . Seventeen up-regulated and seven down-regulated protein spots were identified and are summarized in Table 1. The pIs of the protein spots ranged from 4.32 to 11.07 , and the molecular masses ranged from 10.6 to $95.9 \mathrm{kDa}$. The theoretical and observed $\mathrm{MW} / \mathrm{pI}$ values of the identified proteins were compared. A discrepancy between theoretical and observed $\mathrm{MW} / \mathrm{pI}$ values was shown in our proteomics analysis. This discrepancy is a frequent phenomenon in proteome research and was mainly caused by post-translational modifications of proteins, such as proteolytic processing, glycosylation, and phosphorylation, or it was also possible that the protein spots detected were just protein fragments ${ }^{7,8}$. As shown in Table 1, the expression of 24 proteins was significantly different between the shuffled versus the parent strains. The differentially expressed proteins are classified into four categories: cellular metabolism and energy production (spots $2,3,4,7,8,9,10,11,12$, $13,14,15,18,20,22,23$, and 24); DNA replication, RNA synthesis, and translation (spot 1); posttranslational modification, protein turnover, and chaperones (spots 5, 6, 16, and 19); and hypothetical proteins of unknown function (spots 17 and 21).

Verification of protein expression by qRT-PCRs. Nine proteins with the greatest difference in expression between the parent and shuffled strains were verified by qRT-PCR. Glyceraldehyde-3-phosphate dehydrogenase, enolase, and 6-phosphogluconolactonase are key enzymes of central metabolism and crucial for growth. ATP synthase and $\mathrm{NADH}$ dehydrogenase mediate the balance of energy and oxidation-reduction, which is closely related to the stress response. Secretory protein transports substances and mediates the interactions between cells and the environment. Methylmalonyl$\mathrm{CoA}$ is an important precursor of PA; thus, methylmalonyl-CoA epimerase may affect PA accumulation. These proteins most likely promote acid tolerance by modulating $P$. acidipropionici metabolism. The genes included glyceraldehyde-3-phosphate

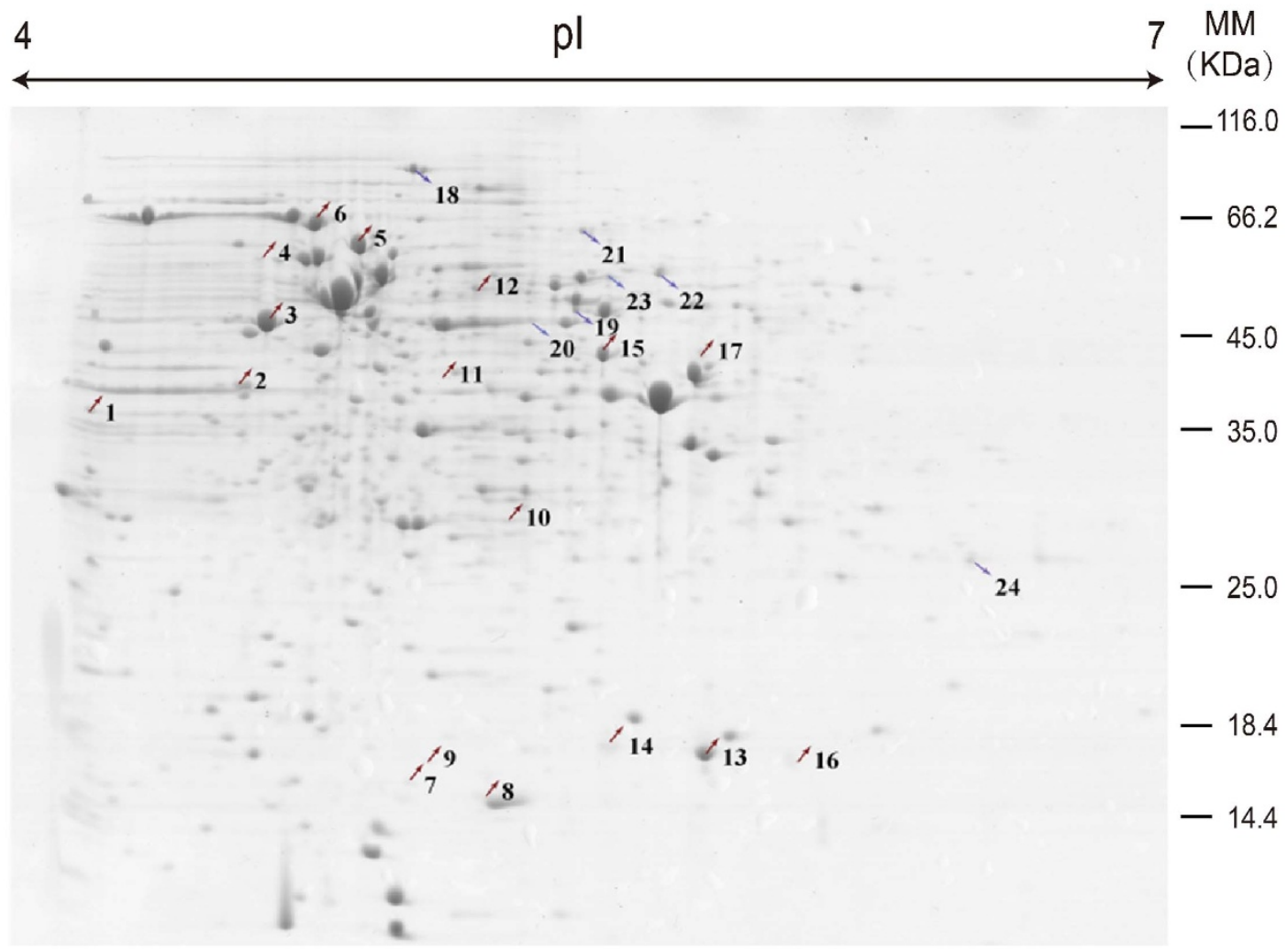

Figure $1 \mid$ Comparative proteome map of $P$. acidipropionici CGMCC 1.2230 and $P$. acidipropionici WSH1105. (up arrow, the up-regulated proteins; down arrow, the down-regulated proteins.) 


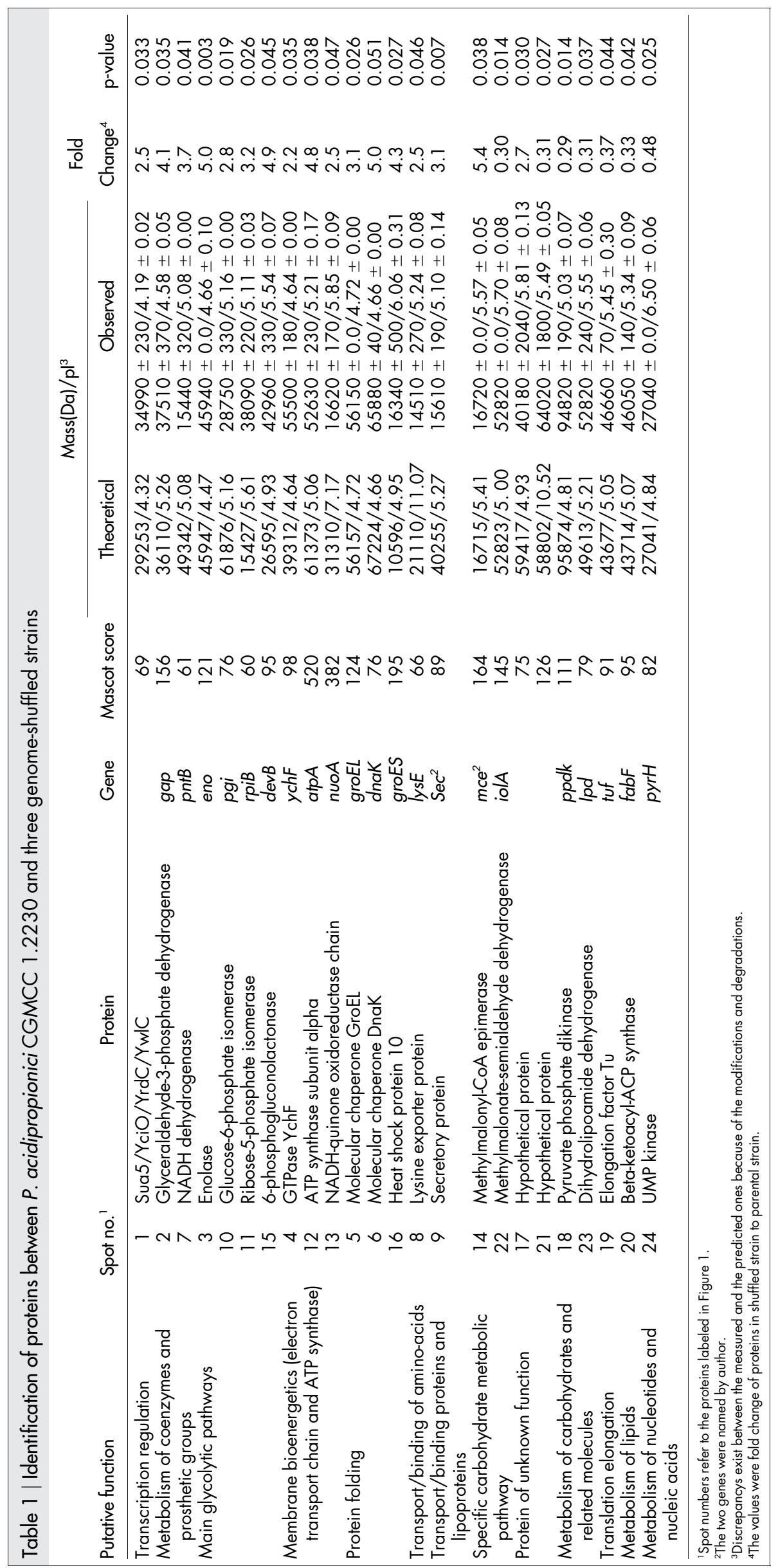




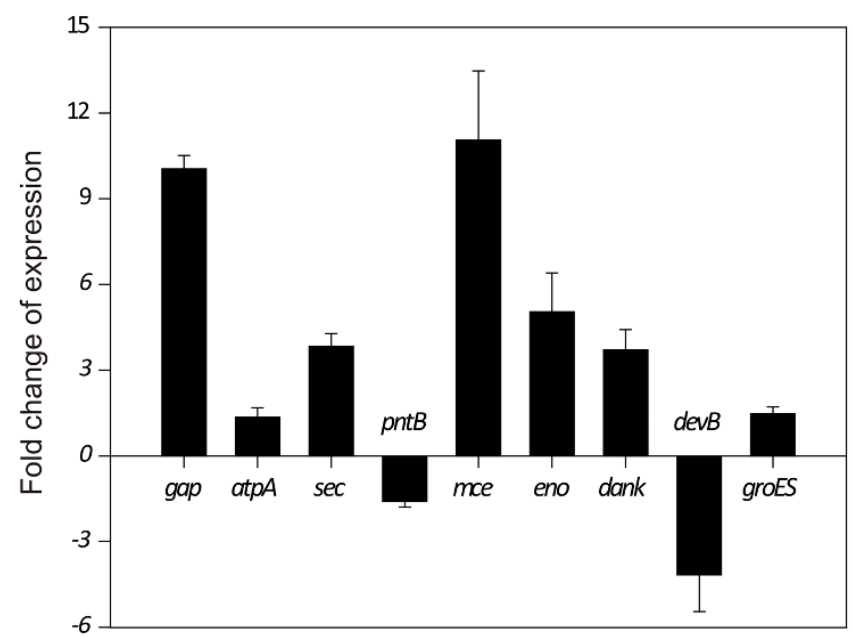

Figure $2 \mid$ Changes of key genes transcription in $P$. acidipropionici WSH1105 compared with the parent strain $P$. acidipropionici CGMCC 1.2230. The values are from three independent cultivations.

dehydrogenase (gap), ATP synthase subunit $\alpha$ (atpA), secretory protein (sec), NADH dehydrogenase (pntB), methylmalonyl-CoA epimerase (mce), enolase (eno), molecular chaperone Dnak (dank), 6-phosphogluconolactonase $(\operatorname{dev} B)$, and heat shock protein GroES (groES). As shown in Figure 2, the transcription levels of 7 genes significantly increased in the shuffled strains, consistent with the proteomics results; however, the transcription levels of $p n t B$ and $\operatorname{dev} B$ decreased in the shuffled strains, suggesting that the strains regulated the accumulation of $\mathrm{NADH}$ dehydrogenase and 6phosphogluconolactonase at the level of translation not transcription.

Overexpression of key proteins in E. coli BL21(DE3) to validate their function in acid tolerance. In order to examine the function of the identified proteins in acid tolerance, we developed the recombinant E. coli strains Ec-g (expressing glyceraldehyde-3phosphate dehydrogenase), Ec-a (ATP synthase subunit $\alpha$ ), Ec-s (secretory protein), Ec-p (NADH dehydrogenase), and Ec-m (methylmalonyl-CoA epimerase) and investigated their expression and effect on acid tolerance. Protein expression was estimated by measuring band intensity after separation by SDS-PAGE.

Acid tolerance was determined by viable-cell counting (Table 2, Figure S2). Ec-s exhibited the greatest tolerance to PA and acetic acid. In culture with $1 \mathrm{~g} / \mathrm{L} \mathrm{PA}$, all recombinant strains showed better growth than the control Ec-1 (E. coli BL21(DE3) with pACYCDuet-1). Strains Ec-g and Ec-a survived in 2 g/L acetic acid., and only Ec-s exhibited tolerance to $2 \mathrm{~g} / \mathrm{L} \mathrm{PA}$ and $4 \mathrm{~g} / \mathrm{L}$ acetic acid.

\section{Discussion}

We performed a proteomics analysis of $P$. acidipropionici acid tolerance in wild-type $P$. acidipropionici CGMCC 1.2230 and the shuffled mutant $P$. acidipropionici wsh1105. The 24 differentially expressed proteins are involved in many crucial biological processes, and the abundance of these proteins varied significantly between the acid-tolerant and parent strains, indicating their importance for acid tolerance in $P$. acidipropionici. The detected proteins mainly belong to four functional classes: cellular metabolism and energy production; DNA replication, RNA synthesis, and translation; posttranslational modification, protein folding, and chaperones; and hypothetical proteins of unknown function.

The cell membrane offers first-level protection from various environmental pressures and is the first function to suffer damage in an acidic environment. Therefore, membrane proteins associated with acid tolerance in $P$. acidipropionici are particularly important. $\mathrm{NADH}$-quinone oxidoreductase chain (NuoA) and ATP synthase subunit $\alpha$ (atpA) mediate membrane bioenergetics; their expression increased by 2.5 - and 4.8 -fold in the shuffled strains. As a multiple subunit enzyme complex embedded in the plasma membrane, $\mathrm{NADH}$-quinone oxidoreductase pumps protons across the cytoplasmic membrane at the start of the respiratory chain and connects proton translocation (cytoplasmic to periplasmic phase) to electron transfer (NADH to quinone). The resulting membrane potential is used to drive processes such as ATP synthesis or solute transport ${ }^{9}$. Subunit NuoA is one of the membrane domain subunits that mediates $\mathrm{H}^{+}$translocation. As shown in Table 1, the NADH-quinone oxidoreductase chain (NuoA) was significantly up-regulated in the shuffled strains, improving proton-pump capacity to adapt to the acidic environment.

In studies on P.acidipropionici acid tolerance at the microenvironment level, $\mathrm{H}^{+}$-ATPase, which is involved in proton-translocation, is critical to the maintenance of intracellular $\mathrm{pH}\left(\mathrm{pH}_{\mathrm{i}}\right)^{10}$. The higher energy status in the shuffled strains provided more energy for $\mathrm{H}^{+}$ATPase catalysis, leading to a higher $\mathrm{pH}_{\mathrm{i}}$ than in the parental strain 6 . A 4.8-fold increase in atpA was observed in the shuffled strains, consistent with previous results in which the shuffled strains exhibited higher $\mathrm{H}^{+}$-ATPase activity and greater $\mathrm{H}^{+}$-ATPase synthesis capacity than the parental strain in order to maintain $\mathrm{pH}_{\mathrm{i}}$ homeostasis.

The glycolytic pathway and TCA cycle play important roles in providing metabolic energy and intermediates during growth. Glucose can be converted into glucose-6-phosphate via the permease/glucokinase pathway and further metabolized into pyruvate by the glycolytic pathway (Figure 3 ). The differentially expressed proteins in the shuffled strains include glyceraldehyde-3-phosphate dehydrogenase, enolase, glucose-6-phosphate isomerase, ribose5-phosphate isomerase, 6-phosphogluconolactonase, pyruvate phosphate dikinase, dihydrolipoamide dehydrogenase, and methylmalonyl-CoA epimerase. A 4.1-fold increase in glyceraldehyde3-phosphate dehydrogenase indicated an increase in glycerate-1, 3-phosphate in the shuffled strains, while the levels of other intermediates are essentially the same, including glycerate-3-phosphate. Thus, the 5-fold increase in enolase and the 3.4-fold decrease in pyruvate phosphate dikinase improved the accumulation of

Table 2 | Comparison of the survival rates (\%) between E. coli BL21 with pACYCDuet-1 (Ec-1) and the recombinant strains in propionic acid and acetic acid

\begin{tabular}{lcccc} 
& $1 \mathrm{~g} / \mathrm{L}$ propionic acid & $2 \mathrm{~g} /$ L propionic acid & $2 \mathrm{~g} /$ Lacetic acid & $4 \mathrm{~g} /$ Lacetic acid \\
\hline Ec- -1 & 0.61 & 0.35 & 0.015 & $<0.003$ \\
Ec-c & 0.72 & 0.003 & 0.010 & $<0.003$ \\
Ec-g & 100 & 0.1 & 0.023 & $<0.003$ \\
Ec-a & 100 & 100 & 17 & $<0.003$ \\
Ec-s & 100 & 0.008 & 2.86 & 0.043 \\
Ec-p & 3.57 & $<0.003$ & $<0.003$ & $<0.003$ \\
Ec-m & 7.61 & $<0.003$ & $<0.003$ \\
\hline Each value represents the mean of triple independent measurements, and the deviation from the mean was below 10\%). & &
\end{tabular}

(Each value represents the mean of triple independent measurements, and the deviation from the mean was below $10 \%$.). 


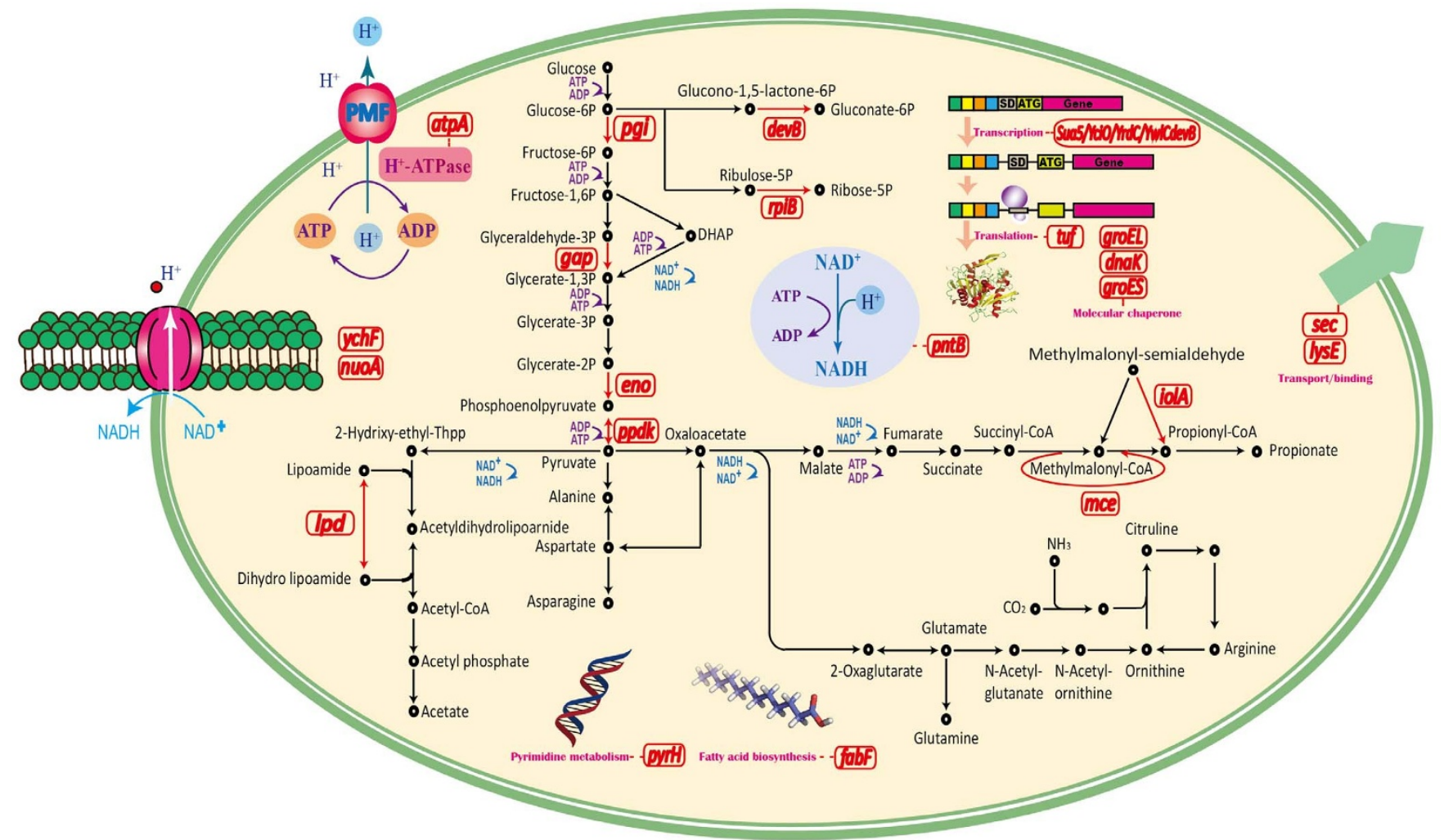

Figure $3 \mid$ Overview of the differentially expressed proteins involved in the metabolism in $P$. acidipropionici.

phosphoenolpyruvate and pyruvate, which are used to generate PA. Methylmalonyl-CoA epimerase (MCE) catalyzes the reversible conversion of (S)-methylmalonyl-CoA to succinyl-CoA, which is a key intermediate in the TCA cycle. It is involved in the metabolism of propionate, branched-chain amino acids, and odd-chain fatty acids, where propionyl-CoA is converted to succinyl-CoA via (R,S)methylmalonyl-CoA ${ }^{11}$. A 5.4-fold increase in MCE may partially explain the increased acid tolerance of the shuffled strains.

Glycolysis produces energy in the form of ATP as well as precursors for anabolic reactions. We previously showed that the metabolism of $\mathrm{NAD}^{+} / \mathrm{NADH}$ is vital for maintaining intracellular $\mathrm{pH}$ homeostasis ${ }^{6}$. Glyceraldehyde-3-phosphate dehydrogenase is a $\mathrm{NAD}^{+} / \mathrm{NADH}$-dependent dehydrogenase that converts glyceraldehyde-3-phosphate to glycerate-1, 3-phosphate in glycolysis. Increased glyceraldehyde-3-phosphate dehydrogenase increases generation of $\mathrm{NADH}$ for reductive biosynthetic reactions. NADH dehydrogenase is directly involved in $\mathrm{NAD}^{+} / \mathrm{NADH}$ metabolism; a 3.7-fold increase in expression could significantly enhance the $\mathrm{NAD}^{+} / \mathrm{NADH}$ ratio and acid tolerance.

During the process of PA fermentation, pyruvate flows into two branches, leading to the formation of propionate or acetate (Figure 3). In the propionate branch of the pathway, $2 \mathrm{NADH}$ molecules are oxidized to $2 \mathrm{NAD}^{+}$; during reduction of pyruvate to acetate, an extra ATP is generated from ADP when acetyl phosphate is converted to acetate, before which acetyl-CoA is converted to acetyl phosphate as the previous step ${ }^{12}$. NADH oxidation can be readily adjusted to need by adjusting the two branches of the pathway, in which the oxidationreduction balance of the cell is preserved. Methylmalonate-semialdehyde dehydrogenase is expressed at a lower level in the shuffled strains than in the parent strain; this enzyme catalyzes the conversion of 2methyl-3-oxopropanoate to propanoyl-CoA and yields $\mathrm{NAD}^{+}$. It also converts 3-oxopropanoate to acetyl-CoA as a participant of the other branch of pyruvate. Thus, the alterations in methylmalonate-semialdehyde dehydrogenase and $\mathrm{NADH}$ dehydrogenase help maintain redox balance during fermentation.
Amino acids are important to the acid tolerance of $P$. acidipropionici. The ADI and GAD acid-resistant systems protect cells against the damaging effects of acidic environments ${ }^{6}$. As central metabolic pathways, the glycolytic pathway and TCA cycle produce precursors for many compounds, including some amino acids. The proteins identified in this study are directly or indirectly involved in amino acid metabolism (Figure 3). As a transport protein, LysE exports Llysine, thereby regulating the intracellular concentration of amino acid. A thorough analysis revealed that LysE is a member of the transmembrane solute translocators ${ }^{13}$. Secretory protein also transports or binds proteins and lipoproteins. To ensure normal cellular activity in an acidic environment, more synthetized proteins need to be removed from the cells. Thus, the 2.5- and 3.1-fold increase in the accumulation of LysE and secretory protein may also enhance acid tolerance. GTPase YchF belongs to a group of universally conserved bacterial GTPases; only a few studies have investigated its function in bacteria. Many bacterial GTPases function by transmitting signals from the ribosome to downstream effectors for specific cellular responses $^{14}$. GTPase YchF of $P$. acidipropionici may play an important role in the regulation of acid tolerance.

Acid stress has a major impact on protein synthesis ${ }^{15}$. Five proteins were associated with stress-response pathways involved in transcription, translation, and protein folding. Under acidic conditions, the DNA repair and protection responses needed for cell survival require the transcription of appropriate genes. Acid exposure in the shuffled strains induced a 2.5 -fold increase in the expression of the transcription regulator Sua5/YciO/YrdC/YwlC. Further research is needed to understand its function in acid tolerance.

Acid exposure also affected the expression of elongation factor $\mathrm{Tu}$ (EF-Tu), which is used to transport aminoacyl tRNAs to the ribosome and to translocate the ribosome down the $\mathrm{mRNA}^{16}$. EF-Tu behaves as a chaperone for unfolded and denatured proteins in $E$. coli, preventing aggregation of citrate synthase during heat shock and producing stable complexes with some unfolded proteins (e.g., reduced carboxymethyl alpha-lactalbumin $)^{18}$. A 2.7 -fold decrease 
in EF-Tu was observed in the shuffled strains, consistent with previous reports that EF-Tu may mediate protein folding and protection during stress in addition to its function in translation elongation ${ }^{17}$.

EF-Tu recognizes the same hydrophobic binding motifs as the chaperone DnaK ${ }^{18}$, which was also induced in the shuffled strains. DnaK in $P$. freudenreichii was reported as a universal chaperone induced by bile salts ${ }^{19}$. The DnaK chaperone machinery prevents misfolding and aggregation of ribosome-bound polypeptides ${ }^{16,20}$ and is part of the heat-shock response-system ${ }^{21}$. DnaK up-regulation under acid stress was observed previously ${ }^{22}$. GroEL and GroES are chaperonins known as heat shock-induced promoters, which are essential for bacterial growth. The GroEL/GroES protein folding chaperonin complex is formed and dissociated by ATP binding and hydrolysis ${ }^{23}$. GroEL assists the folding of stress-denatured polypeptides by actions of binding and encapsulation ${ }^{24}$. It is induced during acid adaptation in $P$. freudenreichii ${ }^{19}$. Proteome analysis showed 3.1- and 4.3-fold up-regulation of GroEL and GroES, respectively, in the shuffled strains. Similar results have been reported in which groEL mRNA expression was increased 2.5-fold by acid shock with change in $\mathrm{pH}$ from 7.0 to $\mathrm{pH} 5.0^{25}$.

Beta-ketoacyl-[acyl-carrier-protein] synthase FabF was 3.0-fold down-regulated in the shuffled strain. Beta-ketoacyl-[acyl-carrierprotein] synthases are necessary for a Type II dissociated fatty acid biosynthetic system. Multiple isoforms of the synthase have been identified; synthase II has a lower $\mathrm{pH}$ optimum and greater resistance to stress ${ }^{26}$. FabF participates in the chain-elongation step of dissociated fatty-acid biosynthesis, during which post-translational modification is required for carrier proteins via phosphopantetheinyl transferase to achieve covalent attachment of the acyl intermediates to acyl carrier protein ${ }^{27}$. FabF regulates fatty-acid composition with changes in growth conditions and is associated with bacterial stress response $^{28}$. Fatty acid distribution has been studied as a common mechanism utilized by $L$. casei to withstand severe acidification and to reduce the deleterious effect of lactic acid on the cell membrane $^{29}$. It seems that microorganisms attempt to maintain normal metabolism in the face of environmental fluctuations by changing the lipid composition of their membranes. Down-regulation of FabF changed membrane fatty acid composition by adjusting the ratio of unsaturated to saturated fatty acids (U/S) and mean chain length, increasing membrane fluidity and permeability of the shuffled strain. This is vital to maintaining homeostasis and effective transmembrane processes such as nutrient transport or solute gradients for energetic purposes.

UMP kinase is specific to uridine monophosphate and is used in the de novo synthesis of pyrimidines. As the reaction it catalyzes is an ATP-consuming process, ATP seems to be economized to reduce the pernicious effects of acid via 2.1-fold down-regulation in the shuffled strains.

Plasmids have not been developed for gene expression in P. acidipropionici; therefore, overexpression of key proteins was conducted in $E$. coli BL21(DE3). Acid tolerance is an important property of $E$. coli, enabling the organism to survive gastric acidity and volatile fatty acids produced in the intestine ${ }^{30}$. E. coli is commonly used to produce various organic acids or other products with organic acid by-products. Thus, many studies have described and improved the acid tolerance mechanisms in E. coli; these studies involved transcriptome analysis, directed evolution, and overexpression and deletion of related proteins ${ }^{31-33}$. Several genes that function in multidrug transporter membrane ATP-binding components, DNA biosynthesis, replication, and repair, transcriptional regulator, and GTPase have been found to serve as acid resistance genes, similar to our findings. In this study, five proteins (glyceraldehyde-3-phosphate dehydrogenase, ATP synthase subunit $\alpha$, secretory protein, NADH dehydrogenase, and methylmalonyl-CoA epimerase) were overexpressed in E. coli BL21(DE3); all yielded varying levels of PA or acetic acid tolerance. Secretory protein overexpression significantly improved the tolerance of E. coli BL21 to PA and acetic acid. E. coli BL21 expressing glyceraldehyde-3-phosphate dehydrogenase and ATP synthase subunit $\alpha$ grew well in low-concentration PA and acetic acid; E. coli overexpressing NADH dehydrogenase and methylmalonyl-CoA epimerase tolerate only $1 \mathrm{~g} / \mathrm{L} \mathrm{PA}$. These improvements in acid tolerance ensure the metabolic processes of $E$. coli will not be inhibited by acidic products, and help cells overcome the damage caused by an acidic environment.

Ours is the first proteomic study of acid tolerance in P. acidipropionici and the differentially expressed proteins involved in many crucial biological processes. qRT-PCR was performed to verify nine proteins that exhibited the greatest differences between the parent and shuffled strains; transcription of seven of these genes significantly increased, consistent with the proteomic findings. Overexpression of five key proteins in E. coli BL21(DE3) validated their function in acid tolerance and the recombinant $E$. coli may have utility as fermentation strains. These results provided insights into the acid-tolerance mechanism of $P$. acidipropionici and enhanced bacterial survival under stress and inform the development of improved PA-producing strains.

\section{Methods}

Microorganisms and culture conditions. The parent strain $P$. acidipropionici CGMCC 1.2230 was purchased from the China General Microbiological Culture Collection (CGMCC). P. acidipropionici WSH1 105 tolerates $20 \mathrm{~g} / \mathrm{L}$ PA was obtained by genome shuffling ${ }^{5}$. The strains were inoculated at $1 \%(\mathrm{v} / \mathrm{v})$ into $100-\mathrm{mL}$ anaerobic jars containing $100 \mathrm{~mL}$ sterile medium $(10 \mathrm{~g} / \mathrm{L}$ yeast extract, $5 \mathrm{~g} / \mathrm{L}$ tryptic soy broth, $1.5 \mathrm{~g} / \mathrm{L} \mathrm{KH}_{2} \mathrm{PO}_{4}$, and $2.5 \mathrm{~g} / \mathrm{L} \mathrm{K}_{2} \mathrm{HPO}_{4}, \mathrm{pH} 7.0$ ). The anaerobic jars were sealed with butyl rubber caps and incubated for $48 \mathrm{~h}$ at $30^{\circ} \mathrm{C}$.

Protein extraction. Proteins were extracted from $15-\mathrm{mL}$ aliquots. Cells were harvested in mid-exponential phase and collected by centrifugation $(10,000 \times g$, $5 \mathrm{~min}$ ). After washing 3 times with ultrapure water, the cells were lysed in $1 \mathrm{~mL}$ buffer containing $7 \mathrm{M}$ urea, $2 \mathrm{M}$ thiourea, 4\% 3-[(3-cholamidopropyl)dimethylammonio]propanesulfonate (CHAPS), and $1 \%$ protease inhibitor, and the solution was sonicated on ice for $40 \mathrm{~min}$, followed by centrifugation at $20,000 \times g$ for $30 \mathrm{~min}$. The proteins in the supernatant were treated with the Clean-up Kit (GE Healthcare) and dissolved in rehydration buffer (7 M urea, $2 \mathrm{M}$ thiourea, 4\% CHAPS, $1 \%(\mathrm{w} / \mathrm{v})$ dithiothreitol (DTT), 0.5\% (v/v) biolytes $\mathrm{pH}$ range 3-10 (Bio-Rad), 0.001\% (w/v) bromophenol blue). Protein concentration was determined using the BioRad Protein Assay Kit (Bio-Rad) with BSA standard.

Two-dimensional gel electrophoresis. Isoelectric focusing (IEF) of the protein samples was performed on immobilized $\mathrm{pH}$ gradient (IPG) strips $(24 \mathrm{~cm}, 4-7 \mathrm{pH}$ linear gradient; Bio-Rad). Samples were diluted to the same concentration with rehydration buffer (400-800 $\mu \mathrm{g}$ protein $/ 450 \mu \mathrm{L})$. IPG strips were passively rehydrated for $14 \mathrm{~h}$ by soaking in $450 \mu \mathrm{L}$ protein samples at room temperature. IEF was performed with Ettan ${ }^{\mathrm{TM}}$ IPGphor ${ }^{\mathrm{TM}} 3$ (GE Healthcare) using a linear ramp program: 0 to $50 \mathrm{~V}$ in $30 \mathrm{~min}, 50$ to $150 \mathrm{~V}$ in $1 \mathrm{~h}, 150$ to $500 \mathrm{~V}$ in $1 \mathrm{~h}, 500$ to $1000 \mathrm{~V}$ in $1 \mathrm{~h}, 1000$ to $2500 \mathrm{~V}$ in $1 \mathrm{~h}, 2500$ to $5000 \mathrm{~V}$ in $3 \mathrm{~h}$, and 5000 to $10000 \mathrm{~V}$ in $3.5 \mathrm{~h}$ with rapid ramping to $90,000 \mathrm{Vhr}$. The temperature was maintained at $20^{\circ} \mathrm{C}$ and the electric current limit was set to $50 \mu \mathrm{A} /$ gel. Before the second dimension, consecutive equilibrations of the gels in equilibration buffer (6 M urea, $2 \%$ sodium dodecyl sulfate (SDS), $0.375 \mathrm{M}$ Tris-HCl, $20 \%$ glycerol, $0.002 \%$ bromophenol blue, $\mathrm{pH} 8.8$ ) containing $0.8 \%(\mathrm{w} / \mathrm{v})$ DTT and $1 \%(\mathrm{w} / \mathrm{v})$ iodoacetamide were performed for $15 \mathrm{~min}$ as suggested by Görg et al. ${ }^{34}$.

Second-dimension electrophoresis was performed on an Ettan DALTsix Electrophoresis System (GE Healthcare). After rinsing in Tris-glycine electrode buffer (25 mM Tris, $192 \mathrm{mM}$ glycine, 0.1\% SDS, pH 8.3), the IPG strips were placed on 1 -mm-thick SDS $(12.5 \%(\mathrm{w} / \mathrm{v}))$ polyacrylamide gels and sealed with $1 \%(\mathrm{w} / \mathrm{v})$ agarose containing a trace of bromophenol blue. SDS electrophoresis was performed first at $2 \mathrm{~W} /$ gel for $90 \mathrm{~min}$, and then at $14 \mathrm{~W} /$ gel until the dye front was $1 \mathrm{~mm}$ from the bottom of the gel. The temperature was maintained at $12.5^{\circ} \mathrm{C}$ with a MultiTemp III system (GE Healthcare).

Staining and image analysis. Gels were fixed in fixation fluid (80\% methanol, $20 \%$ acetic acid) for $30 \mathrm{~min}$ and stained overnight with Coomassie blue $(1.2 \mathrm{~g} / \mathrm{L}$ Coomassie G-250, $100 \mathrm{~g} / \mathrm{L}\left(\mathrm{NH}_{4}\right)_{2} \mathrm{SO}_{4}, 100 \mathrm{~g} / \mathrm{L}$ phosphoric acid, 20\% methanol). Gels were scanned and images captured with Image Master LabScan (GE Healthcare) after washing in ultrapure water until the background was colorless. Three biological duplications for each sample were tested as described. The gel images were analyzed using the PDQuest 8.0.1 software package (Bio-Rad), including image filtering, background subtraction, normalization, and spot detection ${ }^{35}$. Manual determination of spot positions was performed after automatic matching. Spots with quantitative changes (text/control) $>2$ or $<0.5$ were considered. 
Table 3 | Primers used in the qRT-PCR

\begin{tabular}{lccll} 
& Sequence length & Product length & \multicolumn{1}{c}{ Sense Primer $\left(5^{\prime}-3^{\prime}\right)$} & Anti-sense Primer $\left(5^{\prime}-3^{\prime}\right)$ \\
\hline rimM & 552 & 197 & GTGGTGTCCGATGTGCTC & TGCTTGCTTCCCGTGTTG \\
gap & 1005 & 145 & CACTGATGGCGAGAAGGC & GCTGATGATGTGTGCTTGG \\
pntB & 1413 & 125 & TGATCCTCGGTGGCTTCG & GGTGATGATGACGCTCTCG \\
atpA & 1707 & 145 & CACCACCATCGCATCCAG & CTCATTGCCGACCTCTCC \\
sec & 1152 & 183 & GATGCCGCTGTGTTGTCC & CTCGTCGTCGCTGTCAAAG \\
mce & 447 & 134 & CGACGAGGCTTCCAAGTAC & GAACCTGGGTCATGTGCTC \\
eno & 1290 & 192 & CTCAAGGGCGTGCTCAAG & GCCATCGGTGTAGAACTCC \\
dna & 1875 & 153 & ACCACCGACATCAACATCC & CTTGGCGTCCTCAGCAC \\
devB & 744 & 90 & ACGAGGAGTTGACCACAGG & ACAGGCAGAGGTTCACATATTC \\
groES & 297 & 165 & AAGGAGAAGCCGCAGGAG & GTTGAGCAGCAGGTAGTCG \\
\hline
\end{tabular}

Protein identification by mass spectrometry. Spots of interest were excised from the preparative gels and transferred to microtubes. Spots were digested with bovine trypsin (sequencing grade Roche Molecular Biochemicals) using an Ettan ${ }^{\mathrm{TM}}$ digester station (GE Healthcare Life Sciences) as described by Jun et al. ${ }^{36}$, with minor variations. Briefly, spots were washed twice with water and destained by $2 \times 10 \mathrm{~min}$ incubation in $100 \mathrm{mM}$ ammonium bicarbonate and $30 \%$ acetonitrile $(\mathrm{v} / \mathrm{v})$ and dried in a Savant SpeedVac for $30 \mathrm{~min}$. The samples were immersed in $5 \mu \mathrm{L}$ of trypsin $(10 \mathrm{ng} / \mu \mathrm{L})$ in $25 \mathrm{mM}$ ammonium bicarbonate $(\mathrm{pH} 8.0)$ at $4{ }^{\circ} \mathrm{C}$ for $60 \mathrm{~min}$ and the remaining liquid was removed. Then, samples to which $20 \mu \mathrm{L} 25 \mathrm{mM}$ ammonium bicarbonate ( $\mathrm{pH} 8.0)$ had been added were digested overnight at $37^{\circ} \mathrm{C}$. After digestion, $100 \mu \mathrm{L}$ TA60 (mixture containing $60 \% \mathrm{ACN}$ and $0.1 \% \mathrm{TFA}$ ) were added to the spots and merged with the former enzymatic hydrolysate. Finally, the mixture was concentrated to $5 \mu \mathrm{L} ; 1 \mu \mathrm{L}$ of this and $0.4 \mu \mathrm{L}$ of a $3 \mathrm{mg} / \mathrm{mL} \alpha$-cyano- 4 -hydroxytranscinnamic acid matrix in TA85 (mixture containing $85 \%$ ACN and $0.1 \%$ TFA) were spotted onto a MALDI target plate using the dry droplet method for protein identification by mass spectrometry ${ }^{37}$. The peptide fragment ion data acquired from Bruker UltraFlex III MALDI-TOF/TOF mass spectrometer (Bruker Daltonics, Karlsruhe, Germany) were used to search for protein candidates in the NCBInr database through Mascot (Matrix Science) integrated in Biotools (Bruker Daltonics), which is accessible online. Scores calculated by the Mowse scoring algorithm in MASCOT $(p<0.05)$ were considered as positive identifications. The following search parameters were used: the peptide ions and MS/MS tolerance were set to $300 \mathrm{ppm}$ and 0.9 Da respectively; carbamidomethyl and oxidized methionine were set as fixed and variable modifications, respectively, and 1 missed cleavage was allowed. The identified proteins were divided into different categories by the information provided in the NCBInr database.

RNA isolation and quantitative reverse transcription-PCR (qRT-PCR). Cells were harvested in mid-exponential phase and immediately transferred into liquid nitrogen to block the metabolism. Total RNA was extracted using an RNAisoPlus kit (TaKaRa, Dalian, China), and quantitation was performed at $260 / 280 \mathrm{~nm}$ in a Nanodrop ND2000 spectrophotometer (Thermo Scientific, Wilmington, DE, USA). cDNA was synthesized using PrimeScript RT reagent Kit Perfect Real Time (TaKaRa Bio-Inc, Otsu, Shiga Japan). The qRT-PCR was conducted in a LightCycler 480 II Real-time PCR instrument (Roche Diagnostics, Mannheim, Germany) and performed using a SYBR Premix Ex Taq Kit (TaKaRa Bio-Inc, Otsu, Shiga Japan) with a $20.0 \mu \mathrm{L}$ system: $10.0 \mu \mathrm{L}$ SYBR Premix Ex Taq $(2 \times), 0.4 \mu \mathrm{L}$ PCR Forward Primer $(10 \mu \mathrm{M}), 0.4 \mu \mathrm{L}$

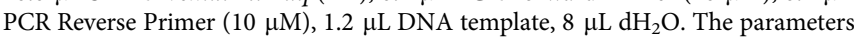
were: pre-incubation at $95^{\circ} \mathrm{C}$ for $30 \mathrm{~s} ; 40$ cycles of amplification step $95^{\circ} \mathrm{C}$ for $5 \mathrm{~s}$, $60^{\circ} \mathrm{C}$ for $20 \mathrm{~s}$; cooling at $50^{\circ} \mathrm{C}$ for $30 \mathrm{~s}$. The qRT-PCR gene-specific primers designed in Primer Express 2.0 (Table 3). 16S rRNA processing protein (rimM) was used as a reference gene for normalization. All experiments were performed with at least three biological replicates.

Plasmid and strain construction. E. coli JM109 was used for plasmid cloning and maintenance; E. coli BL21(DE3) was used as a host for protein expression. LuriaBertani (LB) medium was used for routine culture maintenance. Chloramphenicol $25 \mu \mathrm{g} / \mathrm{mL}$ was added where indicated. Gene manipulations were carried out using standard methods. In P. acidipropionici, gap (lp_1005), atpA (lp_1707), sec (lp_1152, named by author), pntB (lp_1413), and mce (lp_447, named by author) encode glyceraldehyde-3-phosphate dehydrogenase, ATP synthase subunit $\alpha$, secretory protein, NADH dehydrogenase, and methylmalonyl-CoA epimerase, respectively. cpr (lp_1938, $71.5 \mathrm{KDa}$ ), which encodes cytochrome P450 reductase from Catharanthus roseus and is not related to acid stress resistance, was used as the contro $^{38}$. The genes were cloned into pACYCDuet- 1 vector with the T7 promoter. To overexpress glyceraldehyde-3-phosphate dehydrogenase, the gap coding region was PCR-amplified from the genomic DNA of $P$. acidipropionici and cloned into pACYCDuet- 1 at the NcoI and HindIII sites to yield pACYC-gap. pACYC-atpA, pACYC-sec, pACYC-pntB, pACYC-mce, and pACYC-cpr were constructed by inserting atpA between $N d e \mathrm{I}$ and $E c o$ RV, sec between $N c o$ I and HindIII, pntB between NdeI and KpnI, mce between NcoI and NotI, and crp between NcoI and NotI. PCR was performed using primers designed in Primer 5 (Table S1). Gene insertion was verified by restriction mapping, and the absence of undesired mutations introduced during PCR was verified by direct nucleotide sequencing.
Acid tolerance analysis. Viable-cell counts were used to determine whether gene overexpression enhanced the acid tolerance of $E$. coli. For protein expression, overnight pre-inocula were prepared in $\mathrm{LB}$ medium at $37^{\circ} \mathrm{C}$ and transferred to fresh LB at a starting $A_{600}$ of 0.1 and grown to $A_{600} 0.6$. Expression of the recombinant genes was induced by addition of $1 \mathrm{mM}$ IPTG and incubation continued at $30^{\circ} \mathrm{C}$ for an additional $2 \mathrm{~h}$. Cultures were then challenged with $1 \mathrm{~g} / \mathrm{L} \mathrm{PA}, 2 \mathrm{~g} / \mathrm{L}$ PA, $2 \mathrm{~g} / \mathrm{L}$ acetic acid, and $4 \mathrm{~g} / \mathrm{L}$ acetic acid. Incubation continued at $30^{\circ} \mathrm{C}$ to stationary phase and cell survival was analyzed by viable-cell counting. At this stage, the density of the E. coli cultures was about $10^{8}$ cells $/ \mathrm{mL} ; 1 \mathrm{~mL}$ of the same culture was added to $9 \mathrm{~mL}$ distilled water, and several 10 -fold dilutions were made and spread on agar plates. The plates were incubated at $37^{\circ} \mathrm{C}$ and colonies were counted after $12 \mathrm{~h}$.

Statistical analysis. All experiments were performed at least three times, and the results are expressed as the mean \pm standard deviation $(n=3)$. Data were analyzed using the Student's $\mathrm{t}$ test. $\mathrm{P}$ values less than 0.05 were considered statistically significant.

1. Liu, L. et al. Microbial production of propionic acid from propionibacteria: Current state, challenges and perspectives. Crit. Rev. Biotechnol. 32, 374-381 (2012).

2. Suwannakham, S. \& Yang, S. T. Enhanced propionic acid fermentation by Propionibacterium acidipropionici mutant obtained by adaptation in a fibrousbed bioreactor. Biotechnol. Bioeng. 91, 325-337 (2005).

3. Zhang, A. \& Yang, S. T. Propionic acid production from glycerol by metabolically engineered Propionibacterium acidipropionici. Process Biochem. 44, 1346-1351 (2009).

4. Chen, F., Feng, X., Xu, H., Zhang, D. \& Ouyang, P. Propionic acid production in a plant fibrous-bed bioreactor with immobilized Propionibacterium freudenreichii CCTCC M207015. J. Biotechnol. 164, 202-210 (2012).

5. Guan, N. et al. Genome-shuffling improves acid tolerance of Propionibacterium acidipropionici and propionic acid production. Adv. Chem. Res. 15, 143-152 (2012).

6. Guan, N. et al. Systems-level understanding how Propionibacterium acidipropionici respond to propionic acid stress at the microenvironment levels: Mechanism and application. J. Biotechnol. 167, 56-63 (2013).

7. Farley, A. R. \& Link, A. J. Identification and quantification of protein posttranslational modifications. Method Enzymol. 463, 725-763 (2009).

8. Jin, Z. et al. Proteomic analysis of differences in barley (Hordeum vulgare) malts with distinct filterability by DIGE. J. Proteomics 93, 93-106 (2013).

9. Kao, M. C., Bernardo, S. D., Perego, M., Nakamaru-Ogiso, E., Matsuno-Yagi, A. \& Yagi, T. Functional roles of four conserved charged residues in the membrane domain subunit NuoA of the proton-translocating NADH-quinone oxidoreductase from Escherichia coli. J. Biol. Chem. 279, 32360-32366 (2004).

10. Cotter, P. D. \& Hill, C. Surviving the acid test: responses of gram-positive bacteria to low pH. Microbiol. Mol. Biol. R. 67, 429-453 (2003).

11. Han, Y., Hawkins, A. S., Adams, M. W. \& Kelly, R. M. Epimerase (Msed_0639) and Mutase (Msed_0638 and Msed_2055) Convert (S)-MethylmalonylCoenzyme A (CoA) to Succinyl-CoA in the Metallosphaera sedula 3Hydroxypropionate/4-Hydroxybutyrate Cycle. Appl. Environ. Microbiol. 78, 6194-6202 (2012).

12. Len, A. C., Harty, D. W. \& Jacques, N. A. Proteome analysis of Streptococcus mutans metabolic phenotype during acid tolerance. Microbiology 150, 1353-1366 (2004).

13. Vrljic, M. et al. The LysE superfamily: topology of the lysine exporter LysE of Corynebacterium glutamicum, a paradyme for a novel superfamily of transmembrane solute translocators. J. Mol. Microbiol. Biotechnol. 1, 327-336 (1999).

14. Chen, Y. C. \& Chung, Y. T. A conserved GTPase YchF of Vibrio vulnificus is involved in macrophage cytotoxicity, iron acquisition, and mouse virulence. Int. J. Med. Microbiol. 301, 469-474 (2011).

15. Hickey, E. W. \& Hirshfield, I. N. Low-pH-induced effects on patterns of protein synthesis and on internal pH in Escherichia coli and Salmonella typhimurium. Appl. Environ. Microbiol. 56, 1038-1045 (1990). 
16. Len, A. C., Harty, D. W. \& Jacques, N. A. Stress-responsive proteins are upregulated in Streptococcus mutans during acid tolerance. Microbiology 150, 1339-1351 (2004).

17. Caldas, T. D., El Yaagoubi, A. \& Richarme, G. Chaperone properties of bacterial elongation factor EF-Tu. J. Biol. Chem. 273, 11478-11482 (1998).

18. Malki, A., Caldas, T., Parmeggiani, A., Kohiyama, M. \& Richarme, G. Specificity of elongation factor EF-TU for hydrophobic peptides. Biochem. Biophys. Res. Commun. 296, 749-754 (2002).

19. Leverrier, P. et al. Susceptibility and adaptive response to bile salts in Propionibacterium freudenreichii: physiological and proteomic analysis. Appl. Environ. Microbiol. 69, 3809-3818 (2003)

20. Agashe, V. R. \& Hartl, F. U. Roles of molecular chaperones in cytoplasmic protein folding. Semin. Cell. Dev. Biol. 11, 15-25 (2000).

21. Jayaraman, G. C. \& Burne, R. A. DnaK expression in response to heat shock of Streptococcus mutans. FEMS Microbiol. Lett. 131, 255-261 (1995).

22. Jayaraman, G. C., Penders, J. E. \& Burne, R. A. Transcriptional analysis of the Streptococcus mutans hrcA, grpE and dnaK genes and regulation of expression in response to heat shock and environmental acidification. Mol. Microbiol. 25, 329-341 (1997).

23. Tyagi, N. K., Fenton, W. A. \& Horwich, A. L. ATP-triggered ADP release from the asymmetric chaperonin GroEL/GroES/ADP is not the rate-limiting step of the GroEL/GroES reaction cycle. FEBS Lett. 584, 951-953 (2010).

24. Clare, D. K. et al. ATP-triggered conformational changes delineate substratebinding and-folding mechanics of the GroEL chaperonin. Cell 149, 113-123 (2012)

25. Wilkins, J. C., Homer, K. A. \& Beighton, D. Analysis of Streptococcus mutans proteins modulated by culture under acidic conditions. Appl. Environ. Microbiol. 68, 2382-2390 (2002).

26. D’Agnolo, G., Rosenfeld, I. S. \& Vagelos, P. R. Multiple forms of $\beta$-ketoacyl-acyl carrier protein synthetase in Escherichia coli. J. Biol. Chem. 250, 5289-5294 (1975).

27. Worthington, A. S., Rivera Jr, H., Alexander, M. D. \& Burkart, M. D. Mechanismbased protein cross-linking probes to investigate carrier protein-mediated biosynthesis. ACS. Chem. Biol. 1, 687-691 (2006).

28. Tsay, J. T., Rock, C. \& Jackowski, S. Overproduction of beta-ketoacyl-acyl carrier protein synthase I imparts thiolactomycin resistance to Escherichia coli K-12. J. Bacteriol. 174, 508-513 (1992).

29. Wu, C., Zhang, J., Wang, M., Du, G. \& Chen, J. Lactobacillus casei combats acid stress by maintaining cell membrane functionality. J. Ind. Microbiol. Biotechnol. 39, 1031-1039 (2012).

30. Giannella, R. A., Broitman, S. A. \& Zamcheck, N. Influence of gastric acidity on bacterial and parasitic enteric infections: A perspective. Ann. Intern. Med. 78, 271-276 (1973).

31. King, T., Lucchini, S., Hinton, J. C. D. \& Gobius, K. Transcriptomic analysis of Escherichia coli O157:H7 and K-12 cultures exposed to inorganic and organic acids in stationary phase reveals acidulant- and strain-specific acid tolerance responses. Appl. Environ. Microbiol. 76, 6514-6528 (2010).

32. Sandoval, N. R., Mills, T. Y., Zhang, M. \& Gill, R. T. Elucidating acetate tolerance in E. coli using a genome-wide approach. Metab. Eng. 13, 214-224 (2011).
33. Carter, M. Q. et al. Evolutionary silence of the acid chaperone protein HdeB in enterohemorrhagic Escherichia coli O157:H7. Appl. Environ. Microbiol. 78, 1004-1014 (2012).

34. Görg, A. et al. Approach to stationary two-dimensional pattern: Influence of focusing time and immobiline/carrier ampholytes concentrations. Electrophoresis 9, 37-46 (1988)

35. Kolkman, A., Olsthoorn, M. M., Heeremans, C. E., Heck, A. J. \& Slijper, M. Comparative proteome analysis of Saccharomyces cerevisiae grown in chemostat cultures limited for glucose or ethanol. Mol. Cell. Proteomics 4, 1-11 (2005).

36. Jun, H., Kieselbach, T. \& Jonsson, L. J. Comparative proteome analysis of Saccharomyces cerevisiae: A global overview of in vivo targets of the yeast activator protein 1. BMC Genomics 13, 230 (2012).

37. Kumarathasan, P., Mohottalage, S., Goegan, P. \& Vincent, R. An optimized protein in-gel digest method for reliable proteome characterization by MALDITOF-MS analysis. Anal. Biochem. 346, 85-89 (2005).

38. Leonard, E., Yan, Y. \& Koffas, M. A. Functional expression of a P450 flavonoid hydroxylase for the biosynthesis of plant-specific hydroxylated flavonols in Escherichia coli. Metab. Eng. 8, 172-181 (2006).

\section{Acknowledgments}

This work was financially supported by 973 Program (2013CB733902), 111 Project (111-2-06), 863 Program (2011AA100905), and a project funded by the Priority Academic Program Development of Jiangsu Higher Education Institutions.

\section{Author contributions}

L.L., G.C.D. and J.C. conceived and designed the experiments; N.Z.G. performed the experiments; H.D.S. and R.C. analyzed the data; N.Z.G., J.H.L. and L.L. wrote the paper.

\section{Additional information}

Supplementary information accompanies this paper at http://www.nature.com/ scientificreports

Competing financial interests: The authors declare no competing financial interests.

How to cite this article: Guan, N. et al. Understanding of how Propionibacterium acidipropionici respond to propionic acid stress at the level of proteomics. Sci. Rep. 4, 6951; DOI:10.1038/srep06951 (2014)

This work is licensed under a Creative Commons Attribution 4.0 International License. The images or other third party material in this article are included in the article's Creative Commons license, unless indicated otherwise in the credit line; if the material is not included under the Creative Commons license, users will need to obtain permission from the license holder in order to reproduce the material. To view a copy of this license, visit http://creativecommons.org/licenses/by/4.0/ 